\title{
Production of a polyclonal antibody against osteogenic protein-1, and its role in the diagnosis of osteoarthritis
}

\author{
Sonam Choden Bhutia $^{1}$, BSc, MSc, Takhelmayum Amumacha $\underline{\text { Singh }}^{1}$, MBBS, MD, Mingma Lhamu Sherpa ${ }^{1}$, MBBS, MD
}

\begin{abstract}
INTRODUCTION Osteoarthritis (OA) is a progressive degenerative disorder of the articular cartilage. Available diagnostic radiography has been poorly associated with the progress and severity of this clinical disease. As osteogenic protein-1 (OP-1) has been identified as a bone morphogenetic protein with a major role in cartilage repair, we aimed to evaluate its potential role in the diagnosis of OA.

METHODS This was an experimental study conducted at the Department of Biochemistry, Sikkim Manipal Institute of Medical Sciences, India. Polyclonal antibodies (i.e. anti-OP-1[f]) were raised against OP-1 in mice, and subsequently used in a sandwich enzyme-linked immunosorbent assay (ELISA) to detect the presence of OP-1 in the synovial fluids of 75 osteoarthritic patients. For the purpose of correlation, the radiographic assessments of the knees of the 75 patients were graded using the Kellgren-Lawrence scoring system.

RESULT The polyclonal antibody (i.e. anti-OP-1[f]) raised against OP-1 was able to detect the presence of OP-1 in the synovial fluids of all the osteoarthritic patients via sandwich ELISA. The level of the OP-1 was found to be much higher than the reference range and correlated positively with the severity of $O A(r=0.24 ; p=0.04)$.

CONCLUSION Our study shows that the polyclonal antibody, anti OP-1(f), could be used for the immunodiagnosis of osteoarthritis via sandwich ELISA.
\end{abstract}

Keywords: osteoarthritis, osteogenic protein-1, sandwich ELISA, synovial fluid

\section{INTRODUCTION}

Osteoarthritis (OA) of the knee is a degenerative joint disease that is predominantly seen in the elderly population. It is mainly caused by the degradation of articular cartilage ${ }^{(1)}$ and is most commonly attributed to imbalances between anabolic and catabolic processes. ${ }^{(2)}$ While the prevalence of $\mathrm{OA}$ in India is reported to be $17.0 \%-60.6 \%$, this range is based on studies conducted in Maharashtra and Amritsar, and not a pan-India study. ${ }^{(3)}$

Although radiography is the usual method for diagnosing $\mathrm{OA}$, it has been shown to have a poor association with the clinical features of $O A{ }^{(4,5)}$ A simpler and more sensitive means of diagnosing $\mathrm{OA}$ is required. Cartilage-derived molecules present in the synovial fluid may act as a marker of the biosynthetic or degradative changes that occur during OA. ${ }^{(6)}$ Recently, osteogenic protein-1 (OP-1), a member of the bone morphogenetic protein (BMP) family, has shown a great potential for cartilage repair due to its anabolic and anticatabolic effects on cartilage. ${ }^{(7)}$ The cost of producing OP-1 is one of the major barriers for its use in the management of OA. ${ }^{(8)}$ With knowledge regarding the beneficial effects of OP-1 in cartilage regeneration and repair, it has become important to be able to estimate the concentration of OP-1 in osteoarthritic patients in a simple and feasible manner. In the present study, we attempted to isolate OP-1 from the synovial fluid of osteoarthritic patients. The isolated OP-1 was then used to immunise mice intraperitoneally for the purpose of producing polyclonal antibodies (anti-OP-1 [f] immunoglobulin G [lgG]) able to detect the antigenic OP-1 in osteoarthritic patients by sandwich enzyme-linked immunosorbent assay (ELISA).

\section{METHODS}

The present study was conducted in the Department of Biochemistry, Sikkim Manipal Institute of Medical Sciences (SMIMS), Gangtok, Sikkim, India. It was approved by the Institutional Ethics Committee of the same institute.

A total of 75 clinically diagnosed patients (age $40-80$ years) with primary knee osteoarthritis were selected from patients attending Sir Thutop Namgyal Memorial Hospital and Central Referral Hospital, Gangtok. Patient selection was done according to the criteria described by the American College of Rheumatology. ${ }^{(9)}$ Patients who had diabetes mellitus, hypertension, or any systemic disease or localised joint disorders other than OA were excluded from the study. Written informed consent was obtained from all patients who participated in the present study. The synovial fluid collected from the 75 patients was aspirated and centrifuged to remove debris, divided into aliquots, and stored at $-80^{\circ} \mathrm{C}$. Disease severity was assessed and graded from I to IV using the Kellgren-Lawrence $(\mathrm{KL})$ classification system. ${ }^{(10)}$

The highest OP-1 concentration among the 75 clinically diagnosed osteoarthritic patients was selected by sandwich ELISA. Microtitre plates (Tarson, Kolkata, India) were coated with $100 \mu \mathrm{L}$ of anti-OP-1 (sc-9305; Santa Cruz Biotechnology Inc, Santa Cruz, CA, USA) and incubated for two hours at $37^{\circ} \mathrm{C}$. The wells were washed with $0.05 \%$ Tween-20 (PBS/T) and blocked with $3 \%$ bovine serum albumin (SRL Diagnostics, New Delhi, India). Checkerboard titration was performed with serial dilutions of the sample $(1: 10,1: 100,1: 150)$ and enzyme-antibody conjugate

${ }^{1}$ Department of Biochemistry, Sikkim Manipal Institute of Medical Sciences, Sikkim, India

Correspondence: Dr Mingma Lhamu Sherpa, Professor, Department of Biochemistry, Sikkim Manipal Institute of Medical Sciences, Gangtok 737102, Sikkim, India. mingmals@yahoo.com 
$(1: 200,1: 400,1: 600)$, in order to get the most appropriate result. A total of $100 \mu \mathrm{L}$ of synovial fluid sample was added at a dilution of 1:150 and the plates were incubated for two hours. The plates were washed five times before $100 \mu \mathrm{L}$ of anti-OP-1 conjugated with horseradish peroxidase (HRP) (Bangalore Genie Ltd, Bangalore, India) was added at a dilution of 1:200. The plates were then incubated for two hours. The conjugation of anti-OP-1 with HRP was done using $1 \%$ glutaryldehyde, according to the method described by Avrameas. ${ }^{(11)}$ Colour developed after the addition of the substrate 3,3',5,5'-tetramethylbenzidine (TMB) (Bangalore Genie Ltd, Bangalore, India) and the reaction was stopped after 15 mins via the addition of $30 \mu \mathrm{L}$ of hydrochloric acid. Readings were taken at $450 \mathrm{~nm}$ using an ELISA plate reader (RFCL Ltd, New Delhi, India).

The sample with the highest OP-1 titre was qualitatively analysed using Western blot, according to the procedure described by Towbin et al. ${ }^{(12)}$ The sample was resolved using sodium dodecyl sulphate-polyacrylamide gel electrophoresis (SDS-PAGE) using a 10\% gradient gel, before being transferred onto a nitrocellulose membrane (Bangalore Genie Ltd, Bangalore, India) in a Tris-glycine buffer, $\mathrm{pH} 8.3$, at $25 \mathrm{mV}$ for 1.5 hours. The membrane was cut into individual strips and blocked with $\mathrm{PBS} / \mathrm{T}$, with $0.5 \%$ gelatin at $37^{\circ} \mathrm{C}$ for two hours, followed by four extensive washes with PBS/T. The strips were incubated with HRP conjugated with anti-OP-1 for one hour. Antibody binding was revealed using diaminobenzidine (DAB) (HiMedia, Bangalore, India) and $0.03 \% \mathrm{H}_{2} \mathrm{O}_{2}$ in citrate buffer, $\mathrm{pH}$ 5.0, in a culture tube for about 30 mins. The reaction was stopped by rinsing the strips in water.

SDS-PAGE was performed according to the procedure described by Shapiro et al, ${ }^{(13)}$ stacked at a constant current of $50 \mathrm{mV}$. The electrophoresis run was continued until the tracking dye reached $12 \mathrm{~cm}$ in the separating gel. Molecular weight markers (3 kD-205 kD) (Bangalore Genie Ltd, Bangalore, India) were used for the calibration of the gel. After separation, the gel was cut into two parts - one part was stained with Coomassie ${ }^{\circledR}$ blue R-250 for approximately one hour and then destained; the other part was cut horizontally into 12 equal fractions at $1 \mathrm{~cm}$ intervals and were designated OP-1(a) to OP-1(I). Each of the 12 fractions was mechanically ground and transferred to $5 \mathrm{~mL}$ of $0.05 \mathrm{M}$ sodium phosphate buffer (SPB), $\mathrm{pH} 7.2$; the protein was allowed to elute overnight at $4^{\circ} \mathrm{C} .{ }^{(14)}$ The eluants of the 12 fractions were then lyophilised and dialysed against $0.01 \mathrm{M} \mathrm{SPB}$ overnight. Protein concentrations were estimated using Lowry's method. ${ }^{(15)}$ Sandwich ELISA was performed to check the reading of OP-1 in each of the 12 equal gel fractions at a dilution of 1:600 for the anti-OP-1-HRP-conjugate, and at 1:150 for the sample.

White albino female mice aged 8 weeks and weighing 40-42 g were used for immunisation. The animals were reared in the institutional animal house, SMIMS, as per the guidelines set by the Committee for the Purpose of Control and Supervision of Experiments on Animals. ${ }^{(16)}$ Polyclonal antibodies were raised against the OP-1(f) fraction in mouse ascitic fluid, according to the procedure described by Cheirmaraj et al. ${ }^{(17)}$ Purified OP-1 $(\mathrm{f})$ was used as the immunogen after it had been mixed with complete Freund's adjuvant (CFA) (Bangalore Genie Ltd, Bangalore, India) in the ratio of $4: 1$. The mice were immunised intraperitoneally with high titres of CFA with protein on Days 0, 14, 21, 28 and 35. Each mouse received $195 \mu$ g of the purified protein (OP-1) in five doses. A single dose of $500 \mu \mathrm{L}$ of pristane (2,6,10,14-tetramethyl pentadecane) (HiMedia, Bangalore, India) was administered with the second dose of CFA with protein. The ascitic fluid produced in the peritoneal cavity of the mice was collected at weekly intervals from Day 28 to Day 56 by abdominal tapping. Peritoneal cells and lipids were separated from the ascitic fluid by centrifugation at 3,000 rpm. The presence of anti-OP-1(f) $\operatorname{lgG}$ in the ascitic fluid was detected with indirect ELISA using OP-1 (f) at a dilution of 1:100, and anti-human IgG HRP conjugate (Bangalore Genie Ltd, Bangalore, India) at a dilution of 1:1200.

The immunoglobulins were separated from the ascitic fluid by saturation with 35\%-75\% ammonium sulphate (Merck, Mumbai, India) according to the procedure described by Reddy et al. ${ }^{(18)}$ The precipitate was centrifuged at 8,000 rpm for $15 \mathrm{mins}$ at $4{ }^{\circ} \mathrm{C}$, washed with $40 \%$ ammonium sulphate solution, reconstituted in a small volume of $0.05 \mathrm{M} \mathrm{SPB}$, and dialysed against $0.01 \mathrm{M}$ SPB $(\mathrm{pH} 7.2)$ overnight at $4{ }^{\circ} \mathrm{C}$. The protein concentration was estimated, and the protein was designated as anti-OP-1(f) IgG. The protein was stored at $-20^{\circ} \mathrm{C}$ after the addition of $0.1 \%$ sodium azide (Merck, Mumbai, India) as a preservative.

The OP-1 concentration of the synovial fluids from the 75 osteoarthritic patients was detected with sandwich ELISA, using anti-OP-1(f) and anti-OP-1 (sc-9305) conjugated with HRP. To calculate the concentrations of the OP-1 in the synovial fluids, a standard curve was prepared using a known concentration of OP-1 $(78.4 \mu \mathrm{g} / \mathrm{mL})$ diluted in PBS/T to a concentration ranging from $0 \mathrm{ng} / \mathrm{mL}$ to $250 \mathrm{ng} / \mathrm{mL}$.

Statistical analysis was done using the Statistical Package for the Social Sciences version 16.0 (SPSS Inc, Chicago, IL, USA). The correlation between the concentration of OP-1 in the synovial fluid and the severity of OA was determined using the Spearman's rank correlation coefficient. Comparisons of the OP-1 levels among the different stages of OA by $\mathrm{KL}$ grading were analysed using one-way analysis of variance (ANOVA). Data was expressed as mean \pm standard deviation, and a p-value $<0.05$ was considered statistically significant.

\section{RESULTS}

The OP- 1 concentrations in the synovial fluids of the 75 clinically diagnosed osteoarthritic patients were determined using sandwich ELISA. The highest absorbance was noted for the 1:150 dilution of the sample and the 1:200 dilution of the enzyme-antibody conjugate, using the checkerboard titration described in the Methods section. As sample no. 25 showed the highest concentration of OP-1 $(230 \mathrm{ng} / \mathrm{mL})$, it was selected for use in the subsequent analysis. Western blot was done to confirm the presence of OP-1 (Fig. 1). Among the 12 SDS-PAGE fractions, the OP-1 ( $\mathrm{f})$ fraction had the highest concentration of OP-1 (45 ng/mL), with a molecular weight of about $36 \mathrm{kD}$ (Fig. 2). The protein concentration of the same fraction, estimated using Lowry's 
method, was found to be $78.4 \mu \mathrm{g} / \mathrm{mL}$. This fraction (i.e. OP-1 [f]) was purified and used to immunise the mice.

Approximately $11 \mathrm{~mL}$ of total ascitic fluid was collected from each mouse. The level of IgG antibody collected was estimated using indirect ELISA from Day 28 (absorbance $=0.356$ ) to Day 56 (absorbance = 1.679); the level of antibodies increased from Day 28 to Day 56. IgG antibody concentration could not be determined due to the lack of a standard for anti-OP-1(f) IgG. However, for the purpose of the present study, we assumed that the absorbance was directly proportional to the concentration of anti-OP-1 (f) IgG. Using Lowry's method, the protein concentration of anti-OP-1(f) was $125 \mu \mathrm{g} / \mathrm{mL}$.

The mean OP-1 concentration in the synovial fluid of the 75 osteoarthritic patients, obtained using sandwich ELISA, was $92.8 \pm 39.7 \mathrm{ng} / \mathrm{mL}$. This value is much higher than the normal value of about $50 \mathrm{ng} / \mathrm{mL} .{ }^{(19,20)}$ The concentration of OP-1 observed using commercially available anti-OP-1 and using anti-OP-1(f) IgG gave similar readings among the 75 samples of synovial fluid. According to the KL grading, 10 out of the 75 osteoarthritic patients were diagnosed with grade I OA, 27 with grade II OA, 23 with grade III OA, and 15 with grade IV OA (Table I \& Fig. 3). When the OP-1 levels of the synovial fluid were compared with the radiological $\mathrm{KL}$ grading for $\mathrm{OA}$, a significant positive correlation between OP-1 levels of synovial fluid and OA severity $(r=0.24 ; p=0.04)$ was found. Although the concentration of OP-1 was found to be higher in grades III and IV OA, the difference was not statistically significant $(p>0.05)$.

\section{DISCUSSION}

The presence of endogenous OP-1 in human synovial fluid has been studied. In the present study, the sample with the highest OP-1 concentration among the 75 clinically diagnosed osteoarthritic patients (i.e. sample no. 25) was isolated and used to immunise mice intraperitoneally for the production of polyclonal antibodies (anti-OP-1). Among the 12 SDS-PAGE fractions isolated, the antigenic fraction, OP-1(f), had an approximate molecular weight of $36 \mathrm{kD}$. Previous studies have shown that the molecular weight of OP-1 in osteoarthritic patients was around $18-36$ kD. ${ }^{(19,20)}$

The antibody produced in the present study (i.e. anti-OP-1 [f] $\operatorname{lgG}$ ) was found to be useful for the detection of OP-1 in the synovial fluid of osteoarthritic patients. Through the preparation of standards using a known concentration of OP-1 $(78.4 \mu \mathrm{g} / \mathrm{mL})$, we also attempted to measure the concentration of OP-1 in the

Table I. Comparison between osteogenic protein-1 (OP-1) concentration in the synovial fluids of the 75 patients and osteoarthritis severity, as per Kellgren-Lawrence grading.

\begin{tabular}{lr}
\hline Grade of osteoarthritis & OP-1 $(\mathrm{ng} / \mathrm{mL})^{*}$ \\
\hline Grade I $(n=10)$ & $74.6 \pm 24.8$ \\
Grade II $(n=27)$ & $85.0 \pm 32.5$ \\
Grade III $(n=23)$ & $103.0 \pm 42.5$ \\
Grade IV $(n=15)$ & $103.0 \pm 50.0$ \\
\hline
\end{tabular}

Note: One-way ANOVA p-value $=0.09$. ${ }^{\text {Data }}$ is presented as mean \pm standard deviation.

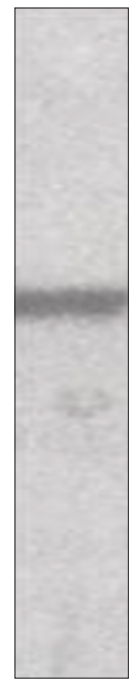

Fig. 1 Image of the Western blot used in the qualitative detection of osteogenic protein-1 using diaminobenzidine substrate.

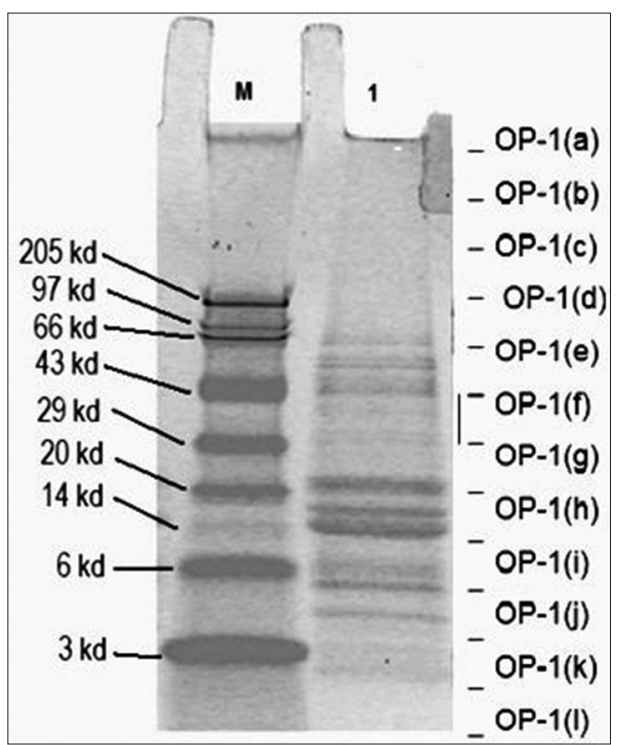

Fig. 2 Image of the SDS-PAGE gel. Lane M: molecular weight markers; lane 1: osteogenic protein-1 (a) to (I).

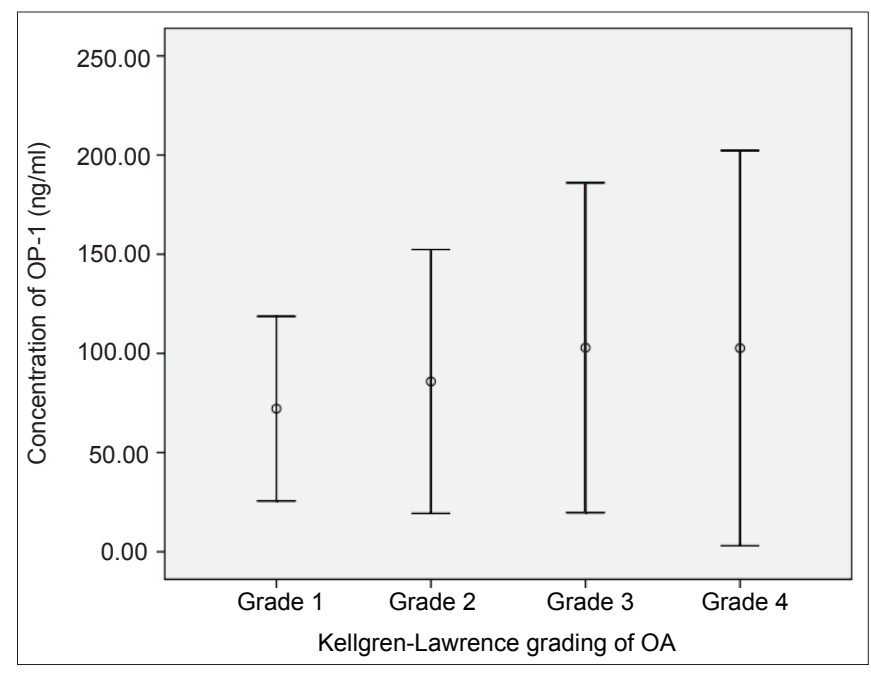

Fig. 3 Graph shows the association between the concentration of osteogenic protein-1 (OP-1) in synovial fluid and the different grades of osteoarthritis (OA), according to the Kellgren-Lawrence grading system. 
synovial fluid of the 75 osteoarthritic patients. We found that the level of the OP-1 in our study cohort was high compared to normal values (i.e. about $50 \mathrm{ng} / \mathrm{mL}$ ). ${ }^{(20,21)}$ An increase in OP-1 levels has also been observed in other studies. ${ }^{(22,23)}$ In the present study, we found a significant positive correlation between the OP-1 level in the synovial fluids of osteoarthritic patients and OA severity, similar to the results of a study by Honsawek et al. ${ }^{(23)}$ This correlation could be due to an increased expression and production of OP-1 in the extracellular matrix, in an autocrine or a paracrine manner. ${ }^{(24)}$ A previous study reported that OP-1 level increases in response to inflammation in OA. ${ }^{(25)}$ In the present study, we found that the OP-1 level in the synovial fluids of patients with grades III and IV OA was higher than in those with grades I and II OA. However, this difference was not statistically significant.

A limitation of the present study is that statistical comparisons between the OP-1 level in synovial fluids from osteoarthritis patients and that in synovial fluids from normal joints could not be done due to ethical reasons. Comparisons using computed tomography and magnetic resonance imaging were also not done due to the unavailability of such facilities. Furthermore, as this was an experimental study with an observational, cross-sectional design, and knee replacement surgery facilities were not available in the country, comparisons of OP-1 levels before and after surgery could not be done. The present study did not analyse the changes in OP-1 levels with exercise, as it was a cross-sectional study.

In conclusion, the present study shows that polyclonal antibodies, such as anti-OP-1(f), can be produced against an antigenic fraction (e.g OP-1[f]) of synovial fluids from osteoarthritic patients. These polyclonal antibodies could be used for the immunodiagnosis of osteoarthritis, as the level of OP-1 correlates positively with $\mathrm{OA}$ severity. Further research studying the differences among the OP-1 levels of synovial fluids from normal joints of different ages and genders, as well as longitudinal studies analysing OP-1 levels before and after treatment, would be required before OP-1 can be used as a marker of OA status and prognosis.

\section{ACKNOWLEDGEMENT}

The authors wish to express their gratitude to the North Eastern Region of Biotechnology Management Programme, DBT India for the grant under the Institutional Level Biotechnology Hub Project.

\section{REFERENCES}

1. Hayashi M, Muneta T, Ju YJ, Mochizuki T, Sekiya I. Weekly intra-articular injections of bone morphogenetic protein-7 inhibits osteoarthritis progression. Arthritis Res Ther 2008; 10:R118.

2. Chambers MG, Bayliss MT, Mason RM. Chondrocyte cytokine and growth factor expression in murine osteoarthritis. Osteoarthritis Cartilage 1997; 5:301-8.
3. Sharma MK, Swami HM, Bhatia V, et al. An epidemiological study of correlates of osteoarthritis in geriatric population of UT Chandigarh. Indian J Community Med 2007; 32:77-8.

4. Lawrence JS, Bremner JM, Bier F. Osteo-arthrosis. Prevalence in the population and relationship between symptoms and x-ray changes. Ann Rheum Dis 1966; 25:1-24.

5. Loeser RF. Age-related changes in the musculoskeletal system and the development of osteoarthritis. Clin Geriatr Med 2010; 26:371-86.

6. Moskowitz R, Goldberg V, Vowell DS, Altman RD, Buckwalter J. Noninvasive markers in osteoarthritis. Osteoarthritis: Diagnosis and Medical/ Surgical Management. 3rd ed. Philadelphia: Saunders, 2001: 293-313.

7. Chubinskaya S, Hurtig M, Rueger DC. OP-1/BMP-7 in cartilage repair. Int Orthop 2007; 31:773-81.

8. Desmyter S, Goubau Y, Benahmed N, de Wever A, Verdonk R. The role of bone morphogenetic protein-7 (Osteogenic Protein-1) in the treatment of tibial fracture non-unions. An overview of the use in Belgium. Acta Orthop Belg 2008; 74:534-7.

9. Altman R, Asch E, Bloch D, et al. Development of criteria for the classification and reporting of osteoarthritis. Classification of osteoarthritis of the knee. Diagnostic and Therapeutic Criteria Committee of the American Rheumatism Association. Arthritis Rheum 1986; 29:1039-49.

10. Kellgren JH, Lawrence JS. Radiological assessment of osteo-arthrosis. Ann Rheum Dis 1957; 16:494-502.

11. Avrameas S. Coupling of enzymes to proteins with glutaraldehyde. Use of the conjugates for the detection of antigens and antibodies. Immunochemistry 1969; 6:43-52.

12. Towbin H, Staehelin T, Gordon J. Electrophoretic transfer of proteins from polyacrylamide gels to nitrocellulose sheets: procedure and some applications. Proc Natl Acad Sci U S A 1979; 76:4350-4.

13. Shapiro AL, Viñuela $E$, Maizel JV Jr. Molecular weight estimation of polypeptide chains by electrophoresis in SDS-polyacrylamide gels. Biochem Biophys Res Commun 1967; 28:815-20.

14. Guellaen G, Goodhardtt M, Hanoune J. Preparative SDS gel electrophoresis. In: J Craigventer, L Harrison, eds. Receptor purification procedures. New York: Alan R Liss, 1984; 2:109-24.

15. Lowry OH, Rosebrough NJ, Farr AL, Randall RJ. Protein measurement with the Folin phenol reagent. J Biol Chem 1951; 193:265-75.

16. Committee for the Purpose of Control and Supervision on Experiments on Animals. CPCSEA Guidelines For Laboratory Animal Facility [online]. Available at: http://icmr.nic.in/bioethics/final_cpcsea.pdf. Accessed November 12, 2012.

17. Cheirmaraj K, Reddy MV, Harinath BC. Diagnostic use of polyclonal antibodies raised in mouse ascitic fluid in bancroftian filariasis. J Immunoassay 1990; 11:429-44.

18. Reddy MV, Prasad GB, Harinath BC. Isolation and evaluation of antigens from microfilaraemia plasma and immune complexes for diagnosis of bancroftian filariasis. Indian J Pathol Microbiol 1986; 29:179-88.

19. Collins DH. The Pathology of Articular and Spinal Diseases. Edward Arnold: London Osteoarthritis, 1949: 74-115.

20. Chubinskaya S, Kumar B, Merrihew C, et al. Age-related changes in cartilage endogenous osteogenic protein-1 (OP-1). Biochim Biophys Acta 2002; 1588:126-34.

21. Merrihew C, Kumar B, Heretis K, et al. Alterations in endogenous osteogenic protein-1 with degeneration of human articular cartilage. J Orthop Res 2003; 21:899-907.

22. Chubinskaya S, Merrihew C, Cs-Szabo G, et al. Human articular chondrocytes express osteogenic protein-1. J Histochem Cytochem 2000; 48:239-50.

23. Honsawek S, Chayanupatkul M, Tanavalee A, et al. Relationship of plasma and synovial fluid BMP-7 with disease severity in knee osteoarthritis patients: a pilot study. Int Orthop 2009; 33:1171-5.

24. Rueger DC, Chubinskaya S. BMPs in articular cartilage repair. In: Vukicevic S, Sampath KT, eds. Bone morphogenetic proteins: regeneration of bone and beyond. Birkhauser: Basel, 2004: 109-32.

25. Chubinskaya S, Frank BS, Michalska M, et al. Osteogenic protein 1 in synovial fluid from patients with rheumatoid arthritis or osteoarthritis: relationship with disease and levels of hyaluronan and antigenic keratan sulfate. Arthritis Res Ther 2006, 8:R73. 